\title{
NOUVELLE
}

\section{La myopathie facio-scapulo-humérale : un défaut de répression?}

Hélène Gilgenkrantz

> La myopathie facio-scapulo-humérale (FSHD), dont l'incidence est de 1 enfant atteint sur 20000 , est la troisième myopathie par ordre de fréquence. Cliniquement, elle se singularise par sa transmission héréditaire dominante et par la distribution variable et asymétrique d'une atteinte musculaire de type dystrophique. Sur le plan moléculaire, elle est remarquable par le mécanisme de l'anomalie génétique longtemps soupçonné et qui vient d'être largement démontré par I'équipe de Rossella Tupler (Pavie, Italie et Boston, USA). Depuis 1992, on savait que le «locus morbide » était situé à l'extrémité du bras long du chromosome 4 [1] et que la mutation caractéristique affectant cette région était une perte plus ou moins importante de matériel génétique portant sur de l'ADN répétitif, succession de motifs identiques de 3500 nucléotides, encore appelés D4Z4 [2, 3]. Ce nombre de répétitions se situe toujours entre 11 et 150 dans la population générale non myopathe, alors qu'il est toujours inférieur à 11 chez les malades atteints de FSHD [4]. Cette anomalie génomique permet, depuis une dizaine d'années, de pratiquer de manière fiable le diagnostic moléculaire et le conseil génétique de la maladie. La sévérité de celle-ci est inversement proportionnelle au nombre de répétitions D4Z4: de 1 à 3 répétitions lorsque la forme est sévère, de 4 à 7 répétitions pour la forme la plus commune et de 8 à 10 répétitions pour la forme mineure.

Cependant, la FSHD demeurait une énigme car la délétion semblait porter sur un territoire pratiquement dépourvu gènes par rapport à des muscles normaux ou atteints d'autres types de myopathies [6]. Certains de ces gènes codent pour des facteurs de transcription musculaires comme MEF2c, connu pour jouer un rôle régulateur essentiel au cours de la myogenèse. Cependant, ces analyses du $\ll$ transcriptome » sont toujours à prendre avec précaution, où la mutation est élucidée mais pas le mécanisme génétique, était tout à fait unique en génétique humaine. Au fil des années, un faisceau d'arguments a suggéré que la région D4Z4 pourrait constituer une zone régulatrice dont la délétion partielle abolirait un mécanisme normal de répression de certains gènes, et induirait une réexpression inappropriée d'une ou plusieurs protéines dans le tissu musculaire. Ce mécanisme pathologique de perte de répression, déjà connu chez la levure, n'avait jamais été décrit chez I'homme. L'hypothèse était difficile à vérifier, car on ne savait pas où chercher le ou les gènes soumis au dérèglement pathologique. Ils pouvaient tout aussi bien se trouver « en cis », à proximité de la région D4Z4 sur le chromosome anormal, ou au contraire « en trans », sur un autre chromosome.

Rossella Tupler et son équipe ont joué un rôle déterminant dans la résolution de cette énigme. Elles ont d'abord montré en 1996 que les individus porteurs d'une très grande délétion emportant un fragment d'ADN plus grand que la région D4Z4 ne présentent aucun signe de FSHD [5]. Cette observation, en faveur de la théorie d'une pathologie de la régulation, suggérait que les gènes potentiellement déréprimés dans le muscle devaient se trouver à proximité des séquences D4Z4 avec lesquelles ils avaient été délétés. Par la suite, cette même équipe a montré que le muscle de sujets atteints de FSHD présente des modifications (positives ou négatives) de l'expression de certains même s'il était alors tentant de spéculer qu'un défaut de transcription de ces gènes était à l'origine de cette affection. L'équipe de Tupler vient à nouveau de franchir une étape décisive dans la compréhension du mécanisme de la FSHD [7]. Elle a tout d'abord montré que trois gènes au moins, FRGl (FSHD region gene 1), FRG2 (FSHD region gene 2) et ANTI (adenine nucleotide translocator1), situés à proximité de la région D4Z4, sont surexprimés dans le muscle des malades, alors qu'ils le sont peu ou pas dans le muscle des témoins normaux. Cette surexpression n'est pas détectée dans les autres tissus et est d'autant plus importante que le nombre de répétitions D4Z4 présenté par les patients est faible. De plus, les auteurs montrent qu'il existe dans la région D4Z4 une séquence capable de se lier à un complexe protéique (D4Z4 repressing complex ou DRC) comprenant notamment le facteur de transcription yyl, la nucléoline et un membre de la famille des protéines nonhistone associées à la chromatine, HMGB2. L'introduction d'oligonucléotides anti-sens ciblant les facteurs yyl, HMGB2 et nucléoline dans des cellules Hela entraîne une surexpression du gène $F R G 2$, indication que les éléments du complexe DRC sont bien impliqués dans la régulation transcriptionnelle de ce gène situé à proximité de D4Z4. On peut donc supposer que, quand la région D4Z4 est intacte, ces facteurs peuvent se fixer et induire une répression transcription- 
nelle de la région adjacente, peut-être par modification de la conformation chromatinienne. A contrario, lors de la délétion caractéristique de la FSHD, cette régulation ne peut s'exercer normalement et les gènes proximaux sont alors surexprimés (Figure 1).

Les implications de ce travail sont multiples. Pour la première fois, une maladie humaine, la FSHD, est associée directement à la dérépression de certains gènes par effet «en cis » de la perte de régions régulatrices constituées par de I'ADN répétitif. Par analogie à la FSHD, d'autres régions répétées VNTR (variable number of tandem repeats), décrites à proximité de locus associés au diabète de type I ou au cancer de la vessie, pourraient également être impliquées dans la physiopathologie de ces affections. Par ailleurs, les résultats obtenus dans la FSHD indiquent des pistes physiopathologiques et des cibles thérapeutiques nouvelles puisque certaines des protéines surexprimées dans le muscle sont déjà identifiées. II faudra élucider leur fonction au cours du développement et, si leur implication dans la pathologie musculaire est démontrée, chercher à empêcher leur expression par des moyens pharmacologiques ou des outils génétiques (interférence ARN). La fonction des gènes $F R G 1$ et $F R G 2$ n'est pas connue. Quant à ANTI, des mutations y ont été retrouvées chez des patients présentant une ophtalmoplégie progressive autosomique dominante $[8,9]$ et une surexpression a été notée dans certaines cardiomyopathies. Son rôle dans la physiopathologie de la FSHD sera donc particulièrement intéressant à suivre, d'autant plus que cette protéine est un composant important du pore mitochondrial de transition et est impliquée dans l'apoptose.

Malheureusement, il est impossible de prévoir aujourd'hui un calendrier des progrès thérapeutiques à venir, d'autant plus que nous manquons cruellement d'un modèle animal expérimental, ce qui oblige pour l'instant à ne travailler que sur des modèles cellulaires.
Sur le plan pratique, le diagnostic moléculaire ne devrait pas être bouleversé par ces données, et l'analyse génomique de la délétion D4Z4 garde tout son intérêt. Néanmoins, la recherche de l'expression ectopique des gènes impliqués dans cette maladie (transcrits et protéines) pourrait devenir un auxiliaire précieux, en particulier dans les cas exceptionnels où, malgré un tableau avéré de FSHD, on ne trouve pas la délétion D4Z4 caractéristique. $\diamond$

Facio-scapulo-humeral atrophy:

a lack of repression?

\section{RÉFÉRENCES}

1. Sarfarazi M, Wijmenga C, Upadhyaya $M$, et al. Regional mapping of facioscapulohumeral muscular dystrophy gene on $4 \mathrm{q} 35$ : combined analysis of an international consortium. Am J Hum Genet 1992; 51: 396-403

2. Wijmenga C, Brouwer OF, Padberg GW, Frants RR.

Transmission of de novo mutation associated with facioscapulohumeral muscular dystrophy. Lancet 1992; 340: 985-6.
3. Wijmenga C, Hewitt JE, Sandkuijl LA, et al. Chromosome 4q DNA rearrangements associated with facioscapulohumeral muscular dystrophy. Nat Genet 1992; 2: 26-30.

4. Lunt PW.

Facioscapulohumeral muscular dystrophy: molecular studies. 44th ENMC International Workshop, 19-21 July 1996, Naarden, The Netherlands. Neuromusc Disord 1998; 8 : 126-30.
5. Tupler R, Berardinelli A, Barbierato L, et al. Monosomy of distal $4 \mathrm{q}$ does not cause facioscapulohumeral muscular dystrophy. J Med Genet 1996; 33: 366-70.

6. Tupler R, Perini G, Pellegrino MA, Green MR. Profound misregulation of muscle-specific gene expression in facioscapulohumeral muscular dystrophy. Proc Natl Acad Sci USA 1999; 96: 12650-4.

7. Gabellini D, Green M, Tupler R. Inappropriate gene activation in FSHD. A repressor complex binds a chromosomal repeat deleted in dystrophic muscle. Cell 2002; 110: 339-48.

8. Kaukonen J, Juselius JK, Tiranti V, et al. Role of adenine nucleotide translocator 1 in mtDNA maintenance. Science 2000; 289: 782-5.

9. Napoli L, Bordoni A, Zeviani M, et al.

A novel missense adenine nucleotide translocator-1 gene mutation in a Greek adPEO family. Neurology 2001; 57: 2295-8.

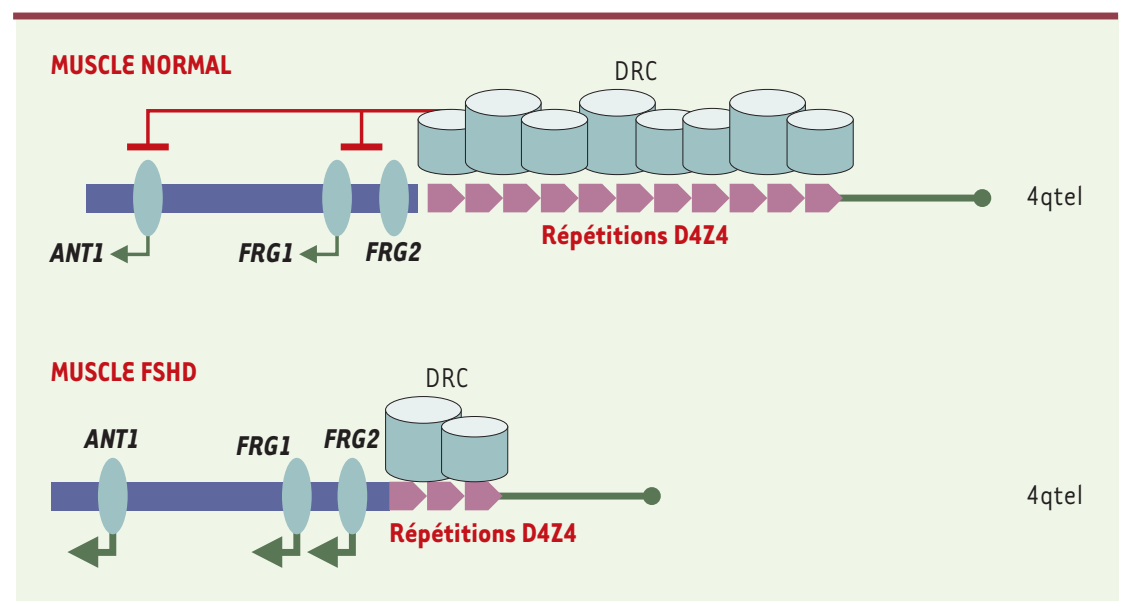

Figure 1. Modèle physiopathologique de la répression génique dans la FSHD. Dans le muscle normal, la présence d'un seuil critique de répétitions D4Z4 permet la répression des gènes de la région (ANT1, FRG1, FRG2) grâce à la fixation d'un complexe multiprotéique (DRC pour D4Z4 repressing complex) qui inhibe leur expression. Dans le muscle de patients FSHD, la délétion d'un certain nombre de répétitions réduit le nombre de complexes répresseurs et entraîne la surexpression inappropriée des gènes adjacents. 